\title{
Evaluation of Dosimetric Performance and Global Uncertainty of the Harshaw 6600 Plus System Used to Staff Monitoring in Côte d'Ivoire
}

\author{
Omer Kouakou1 ${ }^{*}$, Georges Alain Monnehan1,2, Gogon B. D. L. Huberson1 \\ ${ }^{1}$ Laboratoire de physique nucléaire et radioprotection, Unité de Formation et Recherche, Sciences des Structures de la Matière et \\ Technologie, Université Félix Houphouët Boigny, Abidjan, Cote d'Ivoire \\ ${ }^{2}$ Laboratoire National de la Santé Publique; Ministère de la Santé et de l'Hygiène Publique, Abidjan, Cote d'Ivoire \\ Email: *ladjuepe@gmail.com
}

How to cite this paper: Kouakou, O., Monnehan, G.A. and Huberson, G.B.D.L. (2019) Evaluation of Dosimetric Performance and Global Uncertainty of the Harshaw 6600 Plus System Used to Staff Monitoring in Côte d'Ivoire. World Journal of Nuclear Science and Technology, 9, 159-173.

https://doi.org/10.4236/wjnst.2019.94012

Received: July 19, 2019

Accepted: September 8, 2019

Published: September 11, 2019

Copyright (๑) 2019 by author(s) and Scientific Research Publishing Inc. This work is licensed under the Creative Commons Attribution International License (CC BY 4.0).

http://creativecommons.org/licenses/by/4.0/

(c) (i) Open Access

\begin{abstract}
The objective of this work is to check the dosimetric performances of the TLD-100 as stated by the manufacturer as well as the technical standards of radiation protection. The purpose of the performance audit is to assess the inhomogeneity of TLD sensitivity, repeatability and reproducibility, linearity, energy dependence, angular dependence, and fading. All tests were performed under the conditions of ambient temperature and relative humidity recommended by the manufacturer. We began the study by calibrating the Harshaw 6600 Plus, and checking its performance. The TLD-100 performance verification results were all acceptable and in accordance with the manufacturer's advertised values and the radiation protection technical standards. However the performance of the TLD-100 that we have evaluated may have some limitations; these limits, which are sources of uncertainty, have been taken into account in this work by evaluating the overall uncertainty of the $\mathrm{Hp}(10)$ dose in the uncertainty range $9.45 \%$ to $15.80 \%$ by simple formulas. The TLD-100 personal dosimeters and the 6600 Plus reader system indicate that the calculated values of the overall uncertainty $\mathrm{Hp}$ (10) are well below the allowable values of $21 \%$ to $42 \%$ suggested for personal dosimetry services. The obtained data encourage the use of the system for the routine evaluation of the external exposure of workers under ionizing radiation in our laboratory.
\end{abstract}

\section{Keywords}

Overall Uncertainty, Dosimetric Performances, Tests, Dosimetry Services, External Exposure of Workers 


\section{Introduction}

Individual radiological monitoring of workers under ionizing radiation is a regulatory requirement of a radiological protection program [1] that respects the optimization principle [2] [3]. Since 1999, the National Laboratory for Public Health (LNSP), created by decree $\mathrm{n}^{\circ}$ 91-605 of October 09, 1991, through its Subdivision of Protection against Ionizing Radiation (SDPRI), monitors workers' radiation exposure in Ivory Coast [4] using the thermoluminescence technique. The new Harshaw 6600 Plus model dosimetry system offered in 2014 by the International Atomic Energy Agency (IAEA) is based on the phenomenon of thermoluminescence. For accredited dosimetry laboratories, ISO/IEC 17025: 2005 [5] (E) requires an acceptance procedure for any new equipment. Considering that, the creation of the new regulatory authority for radiation protection and nuclear safety (ARSN) through the IVC 6012 project entitled "Establishment of a Secondary Calibration Laboratory and Quality Management" has decided to work according to a quality management approach taking into account the evaluation of the performance of its measurement system [6]. Before the creation of the Laboratory Calibration Calibration and its assessment, this present work carried out under different conditions of irradiation, through dosimetric tests such as the inhomogeneity of the sensitivity of the TLD, the repeatability and the reproducibility, the linearity, energy dependence, angular dependence and fading, involves a quality approach to check the dosimetric performance of TLD-100 as announced by the manufacturer. The sources of erroneous estimation in the evaluation of the measurement of the $\mathrm{Hp}(10)$ doses and the deviations of the operating parameters of the reader [7] have been exploited to evaluate the overall uncertainty of the TLD system for the surveillance of people exposed to ionizing radiation in Côte d'Ivoire.

\section{Materials and Methods}

The Harshaw 6600 Plus dosimetry system developed and produced by Thermo Fisher Scientific (TFS) is composed of thermoluminescent dosimeters (TLDs) in LiF: $\mathrm{Mg}$, $\mathrm{Ti}$, and a reader.

\subsection{Materials}

\subsubsection{Description of TLD-100 Samples}

The dosimeter consists of four chips LiF: Mg, Ti mounted in Teflon on an aluminum card and placed in a plastic holder. The carrier contains a unique filter for each copper, acrylonitrile-butadiene-styrene, Mylar and tin chip. These chips have the property of storing energy received during irradiation and returning it after heating in the form of light. Two of these pellets of size $3.2 * 3.2 * 0.38 \mathrm{~mm}^{3}$ and $3.2 * 3.2 * 0.15 \mathrm{~mm}^{3}$ in the plate make it possible to evaluate respectively equivalent doses at the level of the skin $\mathrm{Hp}(0.07)$ and at the level of the body $\mathrm{Hp}$ (10). Each card has a separate number associated with a barcode allowing for faster backup during playback. 


\subsubsection{Description of the Reader}

The reader of Figure 1 is composed of electronic circuits. During the reading, periodic checks of the intensity of the internal source, the reference light (RL) and the noise of the two photomultiplier tubes (PMT) are done at the beginning and at the end of each new dosimeter group. the procedure used to read the cards is to select the heating parameter using the Winrem analysis software. The heating profile is a preheat to $50^{\circ} \mathrm{C}$ and a linear heating rate of $25^{\circ} \mathrm{C} / \mathrm{sec}$ up to $300^{\circ} \mathrm{C}$, for a total time of 13.3 seconds.

\subsection{Methods}

\subsubsection{Performance Ratings Dosimetry TLD-100}

The reader is calibrated in physical unit $(\mathrm{mSv})$ by irradiating the dosimeters "gold" of calibrations at the Secondary Laboratory of Dosimetry Calibration (LSED) of the Nuclear Research Center of Algiers (CRNA, Algeria), compared to $\mathrm{Hp}(10)$ and Hp (0.07). The reader calibration factor (RCF) and the factors that correct the different sensitivities of the dosimeters (ECC) have been determined. We collected data from the CCT database. Then, in Figure 2, we drawn the frequency curve of the cards of each operational quantity $\mathrm{Hp}(10)$ and $\mathrm{Hp}(0.07)$ in positions (ii) and (iii) of the various pellets as a function of the individual ECCs in order to determine the average of the distribution and the proportion of cards that presents a recurrent ECC.

We found a normal distribution shifted to the left of which $5 \%$ of the cards for the chip in $\mathrm{Hp}(10)$ and $4.5 \%$ for the pellet in $\mathrm{Hp}(0.07)$ have after the calibration of the reader a recurring value of 0 , Mean values and standard deviations of the shifted normal distribution are $0.94 \pm 0.09$ for $\mathrm{Hp}(10)$ and $0.96 \pm 0.1$ for hp (0.07), respectively. From the analysis we have chosen the cards whose ECC were outside the average value and the recurrent value of the distribution that is to say out of range $(0.85-1.03)$. These are the chosen maps that have been experimentally characterized. With the exception of the inhomogeneity of batches of

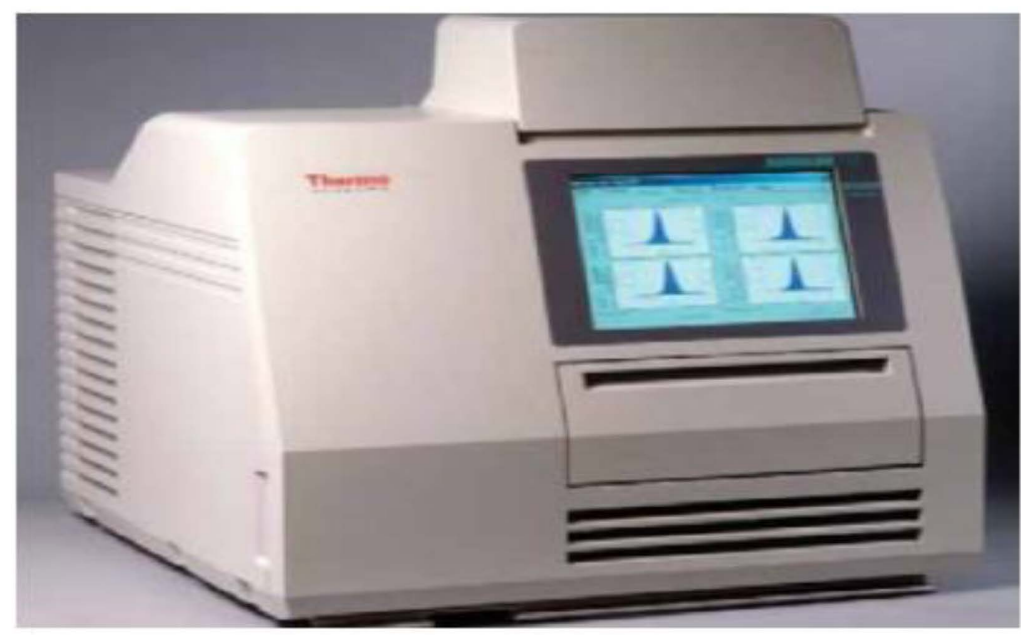

Figure 1. The reader Harshaw 6600 Plus. 


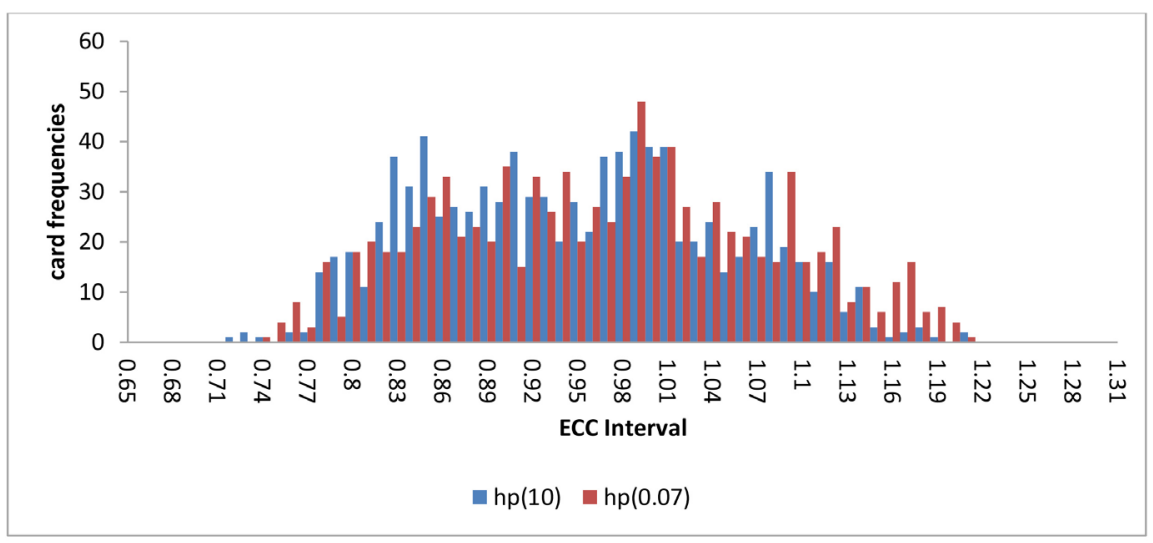

Figure 2. ECC distribution of the total number of cards available at S /DPRI.

dosimeters, Repeatability and Reproducibility carried out using strontium source internal to the reader; all the other irradiations were carried out in different SSDLs during training courses financed by the IAEA. After all the irradiations, the dosimeters with similar fadings [8] are conveyed to be read by the reader.

1) Linearity

We exposed to Cs-137 12 batches of dosimeters; each batch is composed of 4 TLD dosimeters at doses ranging from 0.03 to $15 \mathrm{mSv}$. Exposure time ranges from 1.69 minutes to 20.34 minutes.

2) Inhomogeneity of Batches of Dosimeters

For these measurements, we have, as far as possible, used TLDs from the same batch of our choice. We have irradiated 100 TLD dosimeters of the same batch at an identical dose of $1 \mathrm{mSv}$ from a beam strontium $90 \mathrm{Sr} / \mathrm{Y}$.

3) Repeatability and Reproducibility

Ten (10) exposures of ten (10) $90 \mathrm{Sr} / \mathrm{Y}$ TLD-100 dosimeters were performed under the same conditions. The reference dose is of the order of $1000 \mathrm{gU}$ or $10.83 \mathrm{mSv}$ for an exposure time of $101.7 \mathrm{~s}$ per card. The mean value, standard deviation, and coefficients of variation and responses were determined in Table 1 and Table 2 for each of the ten (10) irradiations and each of the ten (10) dosimeters.

\section{4) Angular Dependence}

The irradiations were carried out in accordance with the reference standards of the national center for radiological protection with a calibrated beam of Cs-137 (OB6 irradiator). We exposed to $2.56 \mathrm{mSv}$ which corresponds to the exposure time of $16 \mathrm{~min}$ a series of eleven batches of dosimeter each consisting of two TL cards irradiated in the same reference position fixed by the lasers. Each lot is exposed in a clearly defined direction. TLD positions for all measures were identical. After each rotation, the geometric center of the detectors is returned. The rotation of the set (phantom + dosimeter) in the clockwise direction assumed to be the positive values of the angles $\left(0^{\circ}, 15^{\circ}, 30^{\circ}, 45^{\circ}, 60^{\circ}, 75^{\circ}\right)$ and counterclockwise 
Table 1. Average $(\bar{x})$, Standard deviation $(\sigma)$, Coefficient of variation $(\mathrm{CV}) \%$, Response for each irradiation.

\begin{tabular}{ccccc}
\hline $\begin{array}{c}\text { Irradiation } \\
\text { (Réproducibility) }\end{array}$ & Average $(\bar{x})$ & $\begin{array}{c}\text { Standard déviation } \\
(\sigma) \%\end{array}$ & $\begin{array}{c}\text { Coefficient of } \\
\text { variation }(\mathrm{CV}) \%\end{array}$ & Response \% \\
\hline 1 & 10.853 & 11 & 1.07 & 100 \\
2 & 10.856 & 11 & 1.04 & 100 \\
3 & 10.743 & 11 & 1.04 & 99 \\
4 & 10.826 & 17 & 1.65 & 99 \\
5 & 10.67 & 12 & 1.13 & 98 \\
6 & 10.823 & 13 & 1.25 & 99 \\
7 & 10.743 & 12 & 1.09 & 99 \\
8 & 10.853 & 12 & 1.15 & 100 \\
9 & 10.751 & 12 & 1.12 & 99 \\
10 & 10.865 & 13 & 1.17 & 100 \\
\hline
\end{tabular}

Table 2. Average $(\bar{x})$, Standard deviation $(\sigma)$, Coefficient of variation $(\mathrm{CV}) \%$, Response for each dosimeter.

\begin{tabular}{ccccc}
\hline $\begin{array}{c}\text { Dosimeter } \\
\text { Repetability })\end{array}$ & Average $(\bar{x})$. & $\begin{array}{c}\text { Standard deviation } \\
(\sigma) \%\end{array}$ & $\begin{array}{c}\text { Coefficient of variation } \\
(\mathrm{CV}) \%\end{array}$ & Response \% \\
\hline 1 & 10.795 & 9 & 0.9 & 99 \\
2 & 10.983 & 7 & 0.6 & 100 \\
3 & 10.679 & 9 & 0.8 & 98 \\
4 & 10.911 & 8 & 0.7 & 99 \\
5 & 10.547 & 6 & 0.6 & 99 \\
6 & 10.827 & 7 & 0.6 & 99 \\
7 & 10.861 & 6 & 0.6 & 98 \\
8 & 10.856 & 7 & 0.6 & 100 \\
9 & 10.757 & 7 & 0.6 & 99 \\
10 & 10.767 & 5 & 0.5 & 100 \\
\hline
\end{tabular}

the negative values $\left(0^{\circ},-15^{\circ},-30^{\circ},-45^{\circ},-60^{\circ},-75^{\circ}\right)$.

5) Energy Dependence

The irradiations were carried out in accordance with the reference standards of the Secondary Laboratory of Dosimetry Calibration (LSED) of the Nuclear Research Center of Algiers (CRNA, Algeria) using three types of beams. An OB6 type emitter emitting a 137Cs gamma beam, a philips radiography apparatus for the $\mathrm{X}$ ray beams, an ELDORADO 78 therapy unit for a 60 Co beam.

6) Thermal Fading

The irradiations were carried out in accordance with the reference standards of the CNESTEN laboratory (Morocco). To highlight the phenomenon of thermal fading, or loss of signal, twenty-four (24) dosimeters were positioned in groups of six (06) on a standardized ghost. They were irradiated with a source of 
cs-137, which emits $\gamma$-rays of $662 \mathrm{keV}$ at a dose of $5 \mathrm{mSv}$ for $32 \mathrm{~min}$. After irradiation, TLDs were stored under ambient temperature conditions $\left(25^{\circ} \mathrm{C}\right)$. Batch readings of four (04) dosimeters were performed at variable times ranging from 24 hours immediately after irradiation to 180 days.

\subsubsection{Assessments of Global Uncertainty}

Two methods of formulating and calculating the overall uncertainty of the TLD system have been applied.

1) Global Method

The first method, called global, is a posteriori estimation of the total uncertainty of the system. It defines two types of uncertainty: The type " $A$ " and the type "B" [9]

Type A, called random involves uncertainties that can in fact be reduced by increasing the number of measurements.

Type B, called systematic is made up of uncertainties that cannot be reduced in number of repeated measures.

It is assumed that the variables to be taken into account follow a uniform or normal statistical distribution. In case the distribution is normal, the Type B uncertainty can be in the form of a standard deviation by dividing the half maximum difference measured by $\sigma_{i}=\frac{\text { maximum measured half difference }}{3}$ [10] where the maximum measured half difference of an amount $X$ is calculated as follows: [10] where the maximum measured half difference of an amount $X$ is calculated as follows:

$$
\text { Maximum measured half difference }=\frac{\operatorname{Max}(X)-\operatorname{Min}(X)}{2}
$$

The overall uncertainty is then:

$$
\begin{gathered}
U_{\text {total système }}=\sqrt{\sum U_{A}^{2}+\sum U_{B}^{2}} \\
U_{\text {total système }}=\sqrt{\sum U_{A}^{2}+\frac{1}{3} \sum \frac{\operatorname{Max}(X)-\operatorname{Min}(X)}{2}}
\end{gathered}
$$

Or $U_{A}$ and $U_{B}$ are the uncertainties of type $A$ and type $B$.

For the calculation of this formula, sources of type $A$ uncertainties are:

○ The Variation of Sensitivity Factors of the Dosimeters (ECC)

- Dosimetric variability (Repeatability and reproducibility of the response) Sources of uncertainty type B are:

○ The irradiation source $90 \mathrm{Sr} / \mathrm{Y}$ obtained by experience

- The calibration factor of the reader provided by laboratory of Algiers

- The electronic parameters of the reader provided by the specification sheet of the manufacturer [11].

- The nonlinearity obtained provides the specification sheet of the manufacturer or experimentally. 
2) Quadratic Summation Method of Each Source of Uncertainty

From formula (2), the dose received by a dosimeter $j$ is evaluated.

$$
H p(10) j=\frac{Q_{j} * E C C_{j}}{R C F}
$$

Or RCF is the calibration factor of the reader, $E C C_{j}$ are the sensitivity factors relative to each dosimeter, $Q_{j}$ is the apparent dose, $H p(10)$ are the actual dose in positions (ii) TLD cards

For the TLD dosimeter, the uncertainty was estimated from the quadratic propagation law of the uncertainties of equation [8].

$$
\sigma_{y}=\sqrt{\sum_{i}^{n}\left(\frac{\partial f}{\partial x_{i}}\right)\left(\sigma_{x_{i}}\right)^{2}}
$$

This formula does not take into account the correlations between the different sources of uncertainty. Variables are assumed to be independent.

From Equation (2), it is possible to identify the different sources of uncertainty of the TLD measure. This gives an expression of the total uncertainty on the TLD measure presented in Equation (4).

$$
\frac{\sigma_{D}}{D}=\sqrt{\sum_{i}^{5}\left(\frac{\sigma_{E C C}}{E C C_{i}}\right)^{2}+\left(\frac{\sigma_{R C F}}{R C F}\right)^{2}+\left(\frac{\sigma_{Q}}{Q}\right)^{2}}
$$

Uncertainty on reading $\sigma_{Q}$

The term $\sigma_{Q}$ corresponds to the uncertainty on the reading. The estimation of this term is mainly based on the results of the study of the characterization of the parameters of performance Equation III summarizes all the uncertainties in reading: It is mainly the most significant influencing factors that have been used to estimate the uncertainty in reading.

With $j=\{$ linearity, inhomogenety, Repeatability and reproducibility, linearity, enegy, angle\}

Or,

$$
\frac{\sigma_{Q}}{Q}=\sqrt{\sum \sigma_{j}^{2}}
$$

Table 3 présents all distributions of uncertainties about reading.

Uncertainty on Ecc sensitivity of TLD $\sigma_{e c c}$ :

$\sigma_{e c c}$ corresponds to the uncertainty about the sensitivity of the TLD. It is given by the builder or experimentation by determining the standard deviation of the Ecc distribution.

Uncertainty about calibration:

$\sigma_{R C F}$ corresponds to the uncertainty on the calibration. This uncertainty was provided by Algeria's secondary calibration laboratory. It takes into account the intrinsic uncertainty and the standard deviation of the measurement performed on cesium-137. 
Table 3. Distribution of all uncertainties on reading.

\begin{tabular}{ccc}
\hline uncertainty $(\%)$ & Type & Distribution \\
\hline$\sigma_{\text {linearity }}$ & B & Normal \\
$\sigma_{\text {inhomogenty }}$ & A & $\begin{array}{c}\text { Expérimental standard déviation of the mean } \\
\text { Expérimental standard déviation of the highest } \\
\text { average enters on repeatability and reproducibility }\end{array}$ \\
$\sigma_{\text {Repetability er réroductibility }}$ & A & Normal \\
$\sigma_{\text {energy }}$ & B & Normal \\
$\sigma_{\text {angle }}$ & B & Normal \\
$\sigma_{\text {fading }}$ & B & \\
\hline
\end{tabular}

Overall uncertainty about the dose measurement of the TLD.

Finally, the total uncertainty on the TLD measure was calculated from the quadratic summation method on the over the low dose range.

\section{Results and Discussions}

\subsection{Performance Ratings Dosimetry TLD-100}

\subsubsection{Linearity}

Figure 3 describes the evolution of the relative response of TLD dosimeters as a function of the dose equivalent $\mathrm{Hp}$ (10). The results were evaluated in terms of Hp (10). The upper line of the curve represents the maximum values of the response relative to the true value and the lower line the minimum values based on the experimental measurements, it is found that most of the results are located inside the trumpet curves. However, many dosimeters are unable to accurately measure in the low dose region. In the low dose range of $0.1 \mathrm{msv}$ to $10 \mathrm{mSv}$, the highest difference between the dose delivered and that measured is $27 \%$. The calculated uncertainty of $4.5 \%$ is below that of the manufacturer. This confirms the common behavior of dosimeters used for occupational exposure monitoring. In the range of doses studied, TLDs respond to IAEA recommendations [12] on linearity. Therefore, it can be concluded that its linearity is sufficient for our use.

\subsubsection{Inhomogeneity of Batches of Dosimeters}

Figure 4 shows the results in the form of dose distributions. The maximum and minimum doses are respectively $1.36 \mathrm{msv}$ and $1.10 \mathrm{msv}$. These values allow us to affirm that some dosimeters receive more dose and others less in relation to the value of exposure. Nevertheless, there is a flat profile, the values are distributed around the average readings of the order of $1.16 \mathrm{msv}$. The calculation shows a standard deviation of the average of the order $6.7 \%$ on the responses of dosimeters of the same batch irradiated under the same conditions. On the other hand all the measured values are beyond the reference value. Standard [13] requires that the limit variation of the maximum and minimum dose relative to the mean value must be less than $30 \%$. According to the manufacturer, this significant variation of $30 \%$ [11] over the entire population of dosimeters chosen is due to the physical mass of the pellets (manufacture). From these series of measurements, 


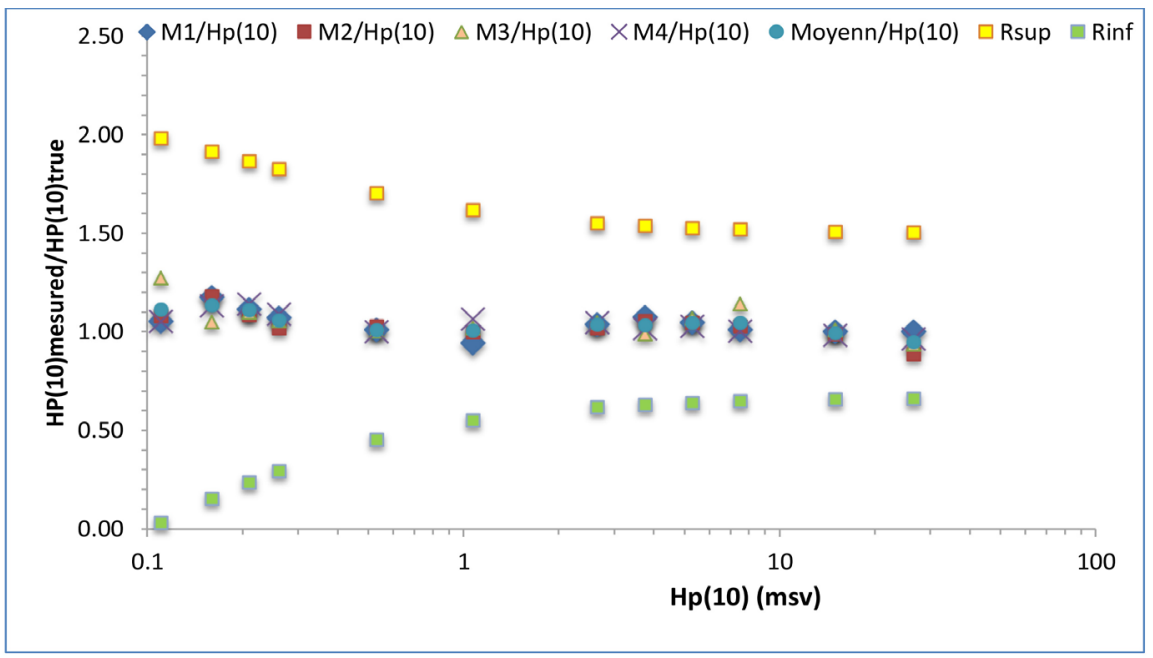

Figure 3. Linearity test according to IAEA recommendations.

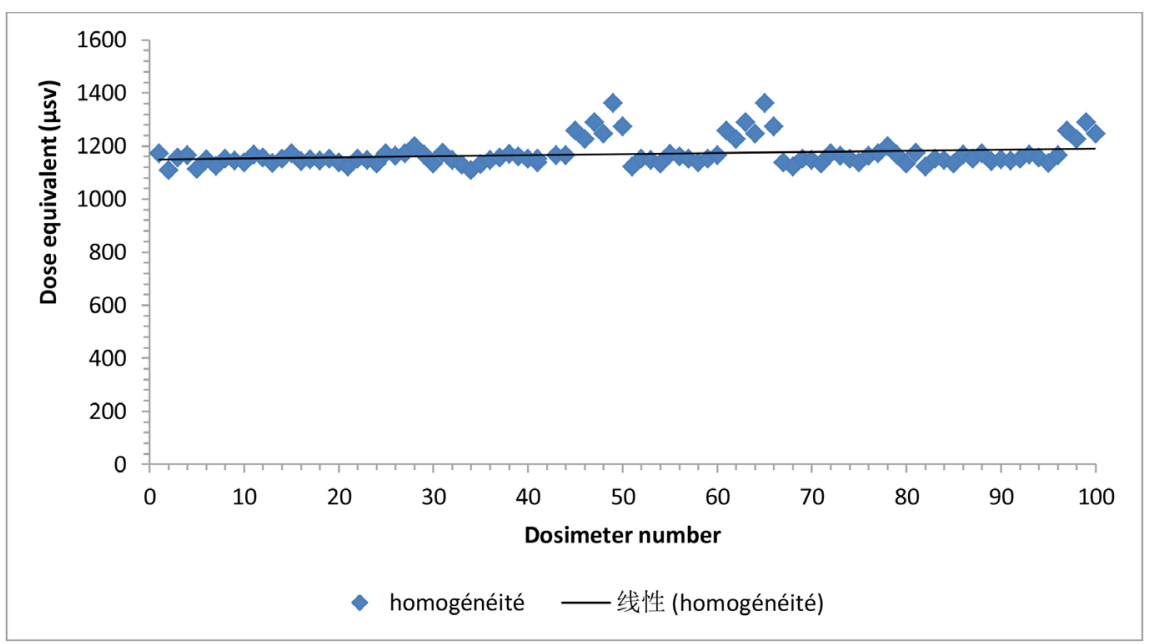

Figure 4. Inhomogeneity test.

we found a value of $22 \%$ which leads us to conclude that the batches of dosimeters used have a good homogeneity and a good stability for evaluation of the doses of routines of the workers exposed to the ionizing radiations.

\subsubsection{Repeatability and Reproducibility}

The repeatability or coefficient of variation is calculated by realizing the ratio of the standard deviation to the average of the measurements. The reproducibility of each irradiation (different dosimeters) is between 98\% - 100\%. These high values are considered good because the system manages to distinguish between dosimeters. The repeatability of each dosimeter is similar only by reasoning on the coefficient of variation for each measurement repeated on the same map it could be said that sometimes happens or the source $90 \mathrm{Sr} / \mathrm{Y}$ still does not put the same dose in the sensitive volume of the tablet when it always carries out the same measurement process because we observe a slight variation of $0.5 \%$ to $0.9 \%$ or a margin of error of $0.4 \%$. 
For each of the 10 irradiations the values obtained are ranged from $1.71 \%$ $2.07 \%$.

For each of the 10 dosimeters the values obtained are ranked from $1.02 \%$ $1.52 \%$.

The coefficients of variation found at each irradiation and each map are below $2 \%$ for all exposures. These values obtained are in line with that of the manufacturer and that of the standard [14] which suggests that the coefficient of variation should not exceed $\pm 5 \%$. The experimental standard deviation of the highest average of $4 \%$ between repeatability and reproducibility was taken as uncertainty.

\subsubsection{Angular Dependence}

Figure 5 shows the responses of the average dose of each lot of normalized dosimeter to the normal incidence value (reference angle). The angles correspond to each measurement point (positioning of the dosimeter). This was evaluated in terms of $\mathrm{Hp}$ dose $(10, \underset{\alpha}{)})$ by rotating the dosimeter-phantom assembly about the vertical axis perpendicular to the direction of incident radiation. We find that the response varies depending on the irradiation angle. The response is maximum at normal incidence but decreases as the ghost rotates. Angles between $15^{\circ}$ and $60^{\circ}$ clockwise and counterclockwise respectively have a relative error range of [ $4 \%-12.7 \%]$ to [ $4 \%-19.4 \%]$. The origin of these considerations could be explained by the fact that, the dosimeter and its case, are not necessarily exposed to direct beams. However, the maximum difference of $12.7 \%$ and $19.4 \%$ observed between responses of two-way TL measurements satisfies the requirements of ISO 4037-3 [15] which states that the difference between responses should not exceed $30 \%$. Further, according to [14] the relative response due to an average energy greater than $65 \mathrm{Kev}$ of the photon radiation and the angle of incidence must be in the range of $-29 \%$ to $67 \%$. Our measurement results are satisfactory. The dosimeters are usable in the range from 00 to 600 . However the detection anisotropy becomes more and more significant from $\pm 75^{\circ}$, where the maximum relative error varies from $62 \%$ to $65.5 \%$. From this inclination the dosimeters do not respond easily.

\subsubsection{Energy Dependence}

Figure 6 shows the ratio of the reading average of four dosimeters and the true conventional value as a function of the photon energy for the $\mathrm{Hp}$ (10) patch at angle of incidence. The responses have been normalized to that of colbat 60 . The normal incidence shows that the variation of the photon energy response for case 8814 in the range of $20 \mathrm{kev}$ to $1250 \mathrm{kev}$ is $-50 \%$ to $+43 \%$. Generally, a good sensitivity of low energy X-rays between 20 and $250 \mathrm{kev}$ is observed, however, the energy dependence is observed for these same $\mathrm{x}$-ray beams or the relative response is between 0.97 and 1.47 compared to that of cesium $(662 \mathrm{KeV})$ and cobalt $(1250 \mathrm{Kev})$. This large difference in response is probably due to the dosimetric system which is not calibrated on X-ray and which does not use an 


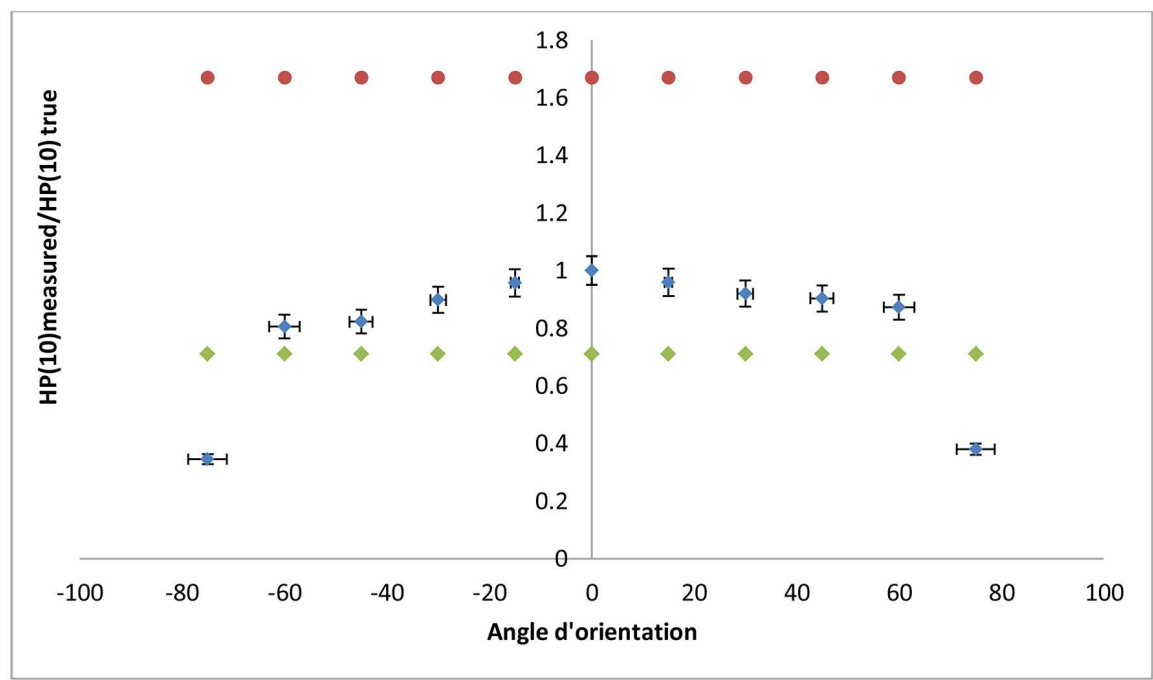

Figure 5. Response as a function of the angle of incidence (positive and negative) of the photons for the Cs-137 source. Values are normalized to $0^{\circ}$ reference angle.

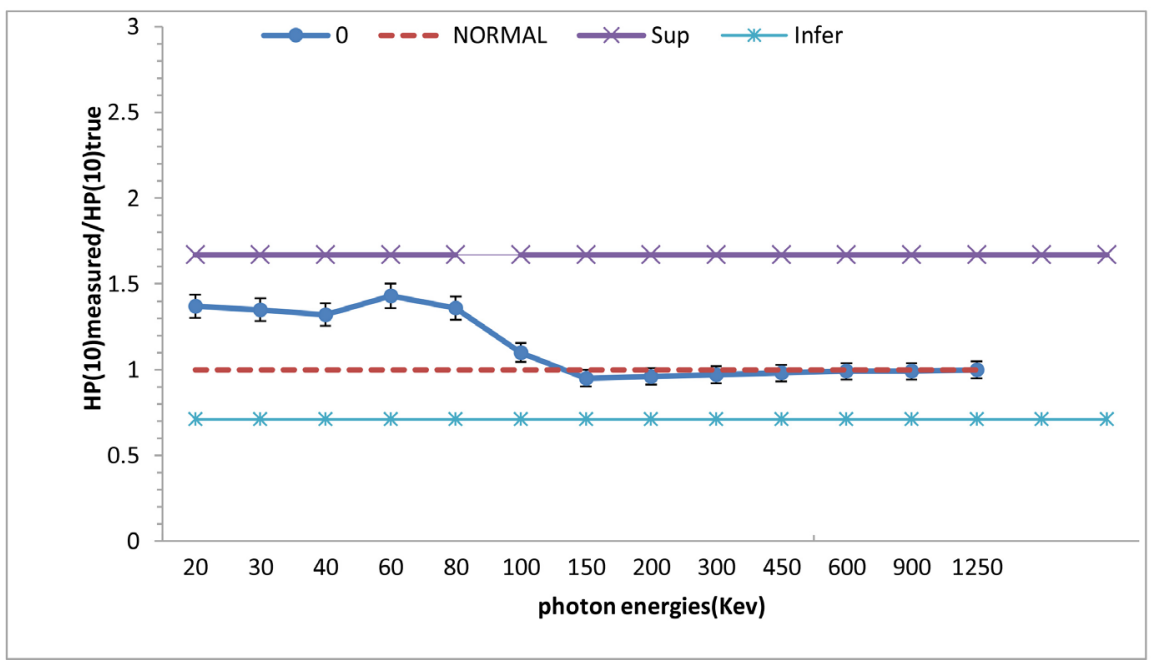

Figure 6. The ratio of the reading average of four dosimeters and the true conventional value as a function of the photon energy for the patch $\mathrm{Hp}(10)$ at $0^{\circ}$ incidence angle. The answers have been normalized to that of colbat 60 .

appropriate dose algorithm incorporating the X-ray beam qualities for the evaluation of $\mathrm{Hp}$ (10) [16] An optimal operating value of the response is found at 80 kev. Nevertheless the relative response of photon energies to the normal incidence compared to the manufacturer [10] and the recommendations of [12] is satisfactory.

\subsubsection{Thermal Fading}

Figure 7 shows the evolution of the actual situation of fading according to the number of days of storage. Each graph of the relative response represents the average reading value of (04) dosimeters.

The signal of the first reading, 24 hours after irradiation, taken as reference is designated $100 \%$ of the luminescence. It is found that the greatest variation 


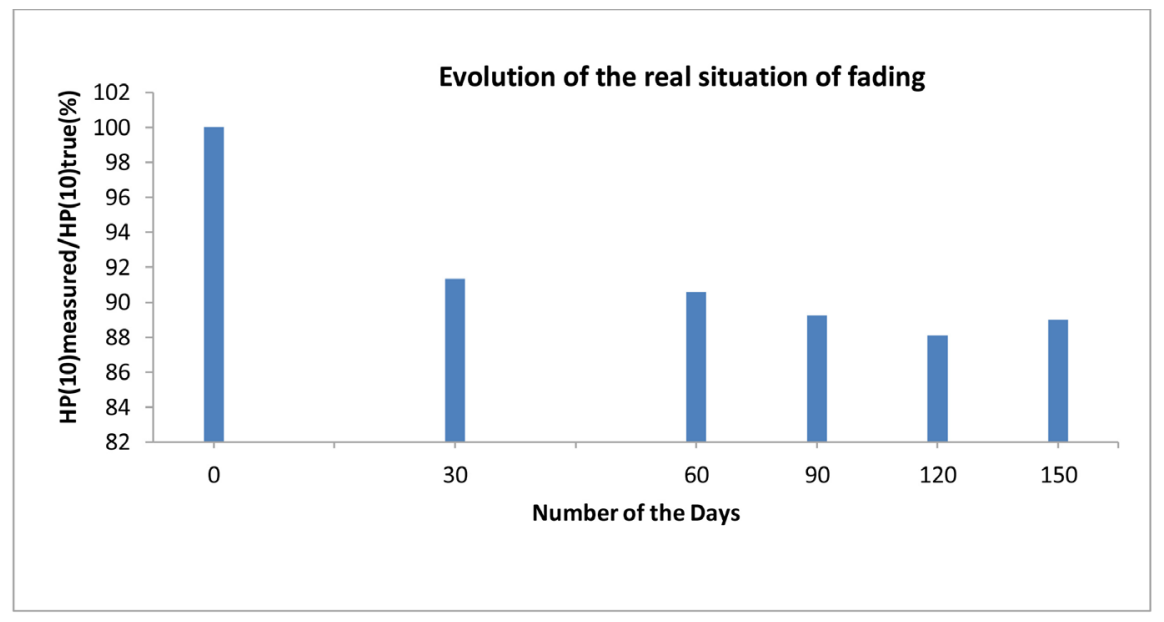

Figure 7. Relative response of the dosimeters as a function of the number of days of storage at room temperature.

occurs during the first 30 days after storage. After 30 days, the fading changes become less progressive, and can reach a correction factor so the average is $10 \%$ - $12 \%$ within 90 days to 150 days. What is very small compared to the characteristics of the manufacturer's material TLD-100 [11]. A significant variation in thermoluminescence was not observed in the 150 days of post-irradiation storage. These measurement results compared to that of the manufacturer and those of [17] [18] show satisfaction.

\subsection{Assessments of Global Uncertainty}

Table 4 and Table 5 show the results of the uncertainties that have been calculated and used in this work.

With regard to Table 4 and Table 5, the uncertainties supposed to be much smaller are the sources of irradiations and the electronic components of the reader since the sources are well calibrated in accordance with the primary standards and the electronic components drift less.

The highest type A uncertainty is the variation in ECCs. This can be reduced by increasing the number of cards to be calibrated. The main source of type B uncertainty is the energy and angle dependency. The overall uncertainty calculated by the two approaches is in the range of $9,45 \%$ to $15 \%, 80 \%$. These values found in our current work are less than $21 \%$ and $42 \%$, values recommended respectively by the ICRP [17] and RSG1.3 [18].

\section{Conclusion}

This work has assessed several parameters, the inhomogeneity of TLD sensitivity, repeatability and reproducibility, linearity, energy dependence, angular dependence and fading as part of our technical quality assurance approach proposed by the laboratory from the ARSN. This assessment made it possible to check the dosimetric performances of the TLD-100 as announced by the manufacturer and the technical standards of radiation protection. We then showed 
Table 4. Assessment of uncertainty from the global method.

\begin{tabular}{cccc}
\hline Source of uncertainty & Type A & Type B & \\
\hline Variation of ECC & $9 \%$ & & \\
Irradiation source & & Reference light & $0.4 \%$ \\
Reader & High tension & $0.005 \%$ \\
& & Heating temperature & - \\
& & PMT electronic noise & - \\
& & linearity & $1 \%$ \\
Total type & $9 \%$ & & $2.5 \%$ \\
Total system & $9.45 \%$ & & \\
\hline
\end{tabular}

Table 5. Assessment of uncertainty from the quadratic method.

\begin{tabular}{|c|c|c|c|}
\hline Source of uncertainty & $\begin{array}{c}\text { Type of } \\
\text { distribution }\end{array}$ & Number of TLDs & $\begin{array}{c}\text { Uncertainty value } \\
\text { Hp (10) (\%) }\end{array}$ \\
\hline Reader Calibration Factor & - & 24 & 2.5 \\
\hline Inhomogeneity of cards & Gaussienne (A) & 100 & 6,7 \\
\hline Repeatability and reproducibility & Gaussienne (A) & 10 & 4 \\
\hline Repeatability of ECC & Gaussienne (A) & 187 & 9 \\
\hline Linearity & Gaussienne (B) & 48 & 4.5 \\
\hline Angular dependence & Gaussienne (B) & 24 & 10 \\
\hline Energy dependence & Gaussienne (B) & 52 & 8 \\
\hline Fading & Gaussienne B) & 24 & 2 \\
\hline Total system & & & 15.80 \\
\hline
\end{tabular}

that the energy and angle dependency is the main source of uncertainty [19]. The overall uncertainty calculated in Table 4 and Table 5 demonstrates that the system complies with the ICRP recommendation on overall accuracy (i.e., an uncertainty range of $-33 \%$ to $+50 \%$ for near-limit doses) and that specified by GSR1.3. Despite the different irradiation conditions, the dosimeters were found satisfactory for the evaluation of the external exposure of workers under ionizing radiation. This work will be refined after the establishment of the secondary calibration laboratory. We can subtract the contribution of natural background radiation and determine the limit of detection.

\section{Conflicts of Interest}

The authors declare no conflicts of interest regarding the publication of this paper.

\section{References}

[1] International Commission on Radiological Protection (1990) Recommendations of 
the International Commission on Radiological Protection. Publication No. 60, Pergamon Press, Oxford and New York.

[2] CIPR 26 (1977) Recommandations 1977 de la Commission Internationale de Protection Radiologique. Pergamon Press, Oxford.

[3] CIPR 60 (1993) Recommandations 1990 de la Commission Internationale de Protection Radiologique. Pergamon Press, Oxford.

[4] Arrêté n 32/MSPS/MEFP du 17 février 1992 (1992) Fixant les modalités de contrôle des activités liées à l'utilisation de sources de rayonnements ionisants et de radioéléments artificiels.

[5] International Organization for Standardization and International Electrotechnical Commission (2005) Conformity Assessment-General Requirements for Bodies Operating Certification of Persons. ISO/IEC 17025. ISO.

[6] Agence Internationale de l'Energie Atomique (2010) Radiation Monitoring \& Protection Services for IAEA Operations. Annual Technical Report, 16.

[7] Luo (2010) Long Term Study of Harshaw TLD LiF-LLD and Uncertainty. Radiation Measurements, 45, 569-572. https://doi.org/10.1016/j.radmeas.2009.11.004

[8] Rizk and Vanhavere, F. (2016) A Study on the Uncertainty for the Routine Dosimetry Service at the Lebanese Atomic Energy Commission Using Harshaw 8814 Dosemeters. Radiation Protection Dosimetry, 170, 168-172.

https://doi.org/10.1093/rpd/ncv426

[9] Joint Committee for Guides in Metrology (2008) Evaluation of Measurement Data-Guide to the Expression of Uncertainty in Measurement. JCGM 100:2008, BIPM.

[10] International Electrotechnical Commission (2015) Radiation Protection Instrumentation, Determination of Uncertainty in Measurement. IEC Technical Report TR 62461. IEC.

[11] Bicron (2002) Model 6600 Automated TLD Reader with WinREMS ${ }^{\mathrm{TM}}$ Operator's Manual. Publication No. 6600-W-O-0602-005. Bicron, Saint-Gobain/Norton Industrial Ceramics Corporation, Solon.

[12] GSR Part 3 (Interim). International Atomic Energy Agency (2011) Radiation Protection and Safety of Radiation Sources: International Basic Safety Standards-Interim Edition. General Safety Requirements Part 3. IAEA Safety Standards Series IAEA.

[13] International Electrotechnical Commission, International Standard (1991) Thermoluminescence Dosimetry Systems for Personal and Environmental Monitoring. CEI IEC 61066, 1991-2012, IEC, Geneva.

[14] International Electrotechnical Commission (2012) Radiation Protection Instrumentation-Passive Integrating Dosimetry Systems for Environmental and Personal Monitoring Part 1: General Characteristics and Performance Requirements. IEC62387-1.

[15] International Organization for Standardization (1993) X and Gamma Reference Radiations for Calibrating Dosemeters and Dose Rate Meters and for Determining Their Response as a Function of Photon Energy. Part 3: Calibration of Area and Personal Dosemeters and the Measurement of Their Response as a Function of Energy and Angle of Incidence. ISO 4037-3. ISO, Geneva.

[16] Arib, M., Herrati, A., Dari, F., Ma, J. and Lounis-Mokrani, Z. (2014) Intercomparison 2013 on Measurements of the Personal Dose Equivalent $\mathrm{hp}(10)$ in Photon Fields in the African Region. Radiation Protection Dosimetry, 163, 276-283. 
https://doi.org/10.1093/rpd/ncu202

[17] Alves, J.G., Abrantes, J.N., Margo, O., Rangel, S. and Santos, L. (2006) Long-Term Stability of a TLD-Based Individual Monitoring System. Radiation Protection Dosimetry, 120, 289-292. https://doi.org/10.1093/rpd/nci520

[18] Schandof, C., Asiamah, A.D. and Anim-Sampong, S. (2002) Performance of Harshaw 6600 Thermoluminescent Dosimeter (TLD), Système for Personal Monitoring. Journal of Applied Science and Technology, 7, 37-43.

[19] Van Dijk, J.W.E. (2011) Developments in Uncertainty Analysis for Individual Monitoring. Radiation Protection Dosimetry, 144, 56-61.

https://doi.org/10.1093/rpd/ncq426 